\title{
Commentaries
}

\section{The intracellular target of butyrate's actions: HDAC or HDON'T?}

Butyrate, the four-carbon short chain fatty acid, has special significance for clinicians and scientists interested in large bowel physiology. It is normally present in the colonic lumen at millimolar concentrations as a product of bacterial fermentation of luminal carbohydrates and is readily taken up by the colonic epithelium to be used as a major energy source via $\beta$-oxidation. Butyrate affects key functions of the colonic epithelium in vivo or at least in vitro in models of the colonic epithelium. These functions include promotion of sodium and water absorption, improvement of tight junction permeability, and acceleration of epithelial restitution. Thus, butyrate plays an important role in the maintenance of colonic mucosal health.

Butyrate has also been implicated in the pathogenesis of colonic diseases, especially colorectal cancer and ulcerative colitis. Butyrate's role in the pathogenesis of ulcerative colitis has been a fascinating saga. In 1981, Roediger first reported that epithelial cells isolated from the rectum of patients with ulcerative colitis exhibited impaired $\beta$-oxidation of butyrate. ${ }^{1}$ His "energy-deficiency" hypothesis created more attention when diversion colitis, which may histologically resemble ulcerative colitis, was shown to be largely caused by a deficient supply of short chain fatty acids in the lumen. ${ }^{2}$ Whether a defect in $\beta$-oxidation has specificity for ulcerative colitis and indeed whether it is more than an in vitro artefact have been questioned, but attempts to resolve these issues have not really succeeded. The report by Ahmad et al (see page 493), in which deficient $\beta$-oxidation of colonic epithelial cells identical to that in ulcerative colitis was shown also to occur in dextran sulphate induced colitis in mice, rekindles debate about this issue. The recent profusion of models of colitis in animals deficient in key immune molecules has tended to draw attention away from the potential primary role of the colonic epithelium in ulcerative colitis. In none of these models, however, do the alterations in crypt architecture at all resemble those that characterise ulcerative colitis. Only models induced by epithelial injury, especially dextran sulphate induced colitis, mimic the shortened and branched crypts of ulcerative colitis, in contrast to the hypertrophic, straight crypts observed in the immune models. We now need information about the metabolic characteristics of the colonic epithelial cells in immune based models of colitis to determine whether the metabolic abnormalities in epithelial cells are indeed secondary to inflammation itself or are specific to the aetiology.

A vexing question has been how this small molecule exerts such a wide array of effects. Butyrate may act indirectly. In cell lines, exposure to butyrate induces a transient intracellular acidification, which itself might trigger many cellular events. Whether this occurs in vivo is uncertain, but would require intermittent rather than continuous exposure to butyrate to occur, conditions more likely to be found in the distal colon. Butyrate may affect cells via the supply of energy from its $\beta$-oxidation. This has been shown in vivo in the atrophic colon starved of short chain fatty acids ${ }^{3}$ and in vitro in a cell line not able to meet its energy needs through other substrates. ${ }^{4}$ However, apart from stimulation of proliferation under energy deficient conditions, the evidence is scanty for a role of $\beta$-oxidation in butyrate's other cellular effects.

Most of butyrate's effects seem to result from a direct action of butyrate itself on intracellular targets. A key target may be histone deacetylase (HDAC). Cells exposed to butyrate exhibit hyperacetylation of core histones, owing to the reversible inhibition of HDAC by butyrate. The importance of butyrate's effect on HDAC has been highlighted by the demonstration that trichostatin A (TSA), which specifically inhibits HDAC, mimics many of the effects of butyrate on specific protein expression, such as interleukin 8 and urokinase receptor, cell proliferation and apoptosis, and epithelial functions, such as paracellular permeability and cell migration. However, Siavoshian and colleagues (see page 507) report that TSA does not mimic butyrate's effect on markers of cell differentiation, specifically the activities of brush border hydrolases, in HT29 cells. This observation has also been recently reported in other colonic epithelial cell lines. ${ }^{5}$ Does this mean that butyrate is acting on hydrolase activities via an intracellular target system that does not involve inhibition of HDAC?

Siavoshian et al examined whether the inhibition of cell proliferation induced by TSA in HT29 cells did truly mimic that of butyrate by comparing their effects on cell cycle events and on key intracellular molecules involved in those events. The effects of butyrate and TSA differed in the changes induced in cyclin dependent kinases and the stage in the cell cycle at which the cells were arrested $\left(G_{1}\right.$ for butyrate, $G_{1}$ and $G_{2}$ for TSA). Furthermore, the duration that histone $\mathrm{H} 4$ was hyperacetylated differed, with TSA having a short action ( $<15$ hours) and butyrate still exerting its effect after 24 hours.

Interpretation of these findings is aided by an understanding of the emerging complexity of HDAC as a transcriptional regulatory system. Histone acetylation precedes transcription and alters nucleosome and chromatin structure. This enhances accessibility of transcription factors to nucleosomal DNA. It is a dynamic process involving two enzyme systems, histone acetyltransferase and HDAC, which catalyse rapid acetylation and deacetylation. HDACs comprise at least two families of proteins that are targeted to specific promoters through sequence specific DNA binding factors. ${ }^{6}$ It seems likely, though not yet proved, that different HDAC proteins will target different promoters and, therefore, subserve different functions. In turn, different inhibitors may exert different spectra of inhibition of HDAC proteins. Siavoshian et al's findings may reflect such an effect, in addition to the different kinetics of their actions. Inhibition of HDAC leads to increased transcription of HDAC mRNA. The recent report that exposure to butyrate or TSA induces differing patterns of mRNA for HDAC proteins ${ }^{7}$ further supports the notion of heterogeneity in the patterns of inhibition of specific HDAC proteins.

Thus, whether HDAC is the major intracellular target for butyrate's actions remains unresolved, but it cannot be assumed that, if TSA does not mimic butyrate's action, then HDAC is not involved. Nevertheless, it seems more likely that butyrate has other intracellular targets, particu- 
larly as, unlike TSA, butyrate probably does not directly bind to HDAC and requires phosphatase activity to exert its inhibitory effect. ${ }^{8}$ Resolution of these issues is eagerly awaited as definition of the molecular pathways by which butyrate acts will greatly improve our understanding of multiple cellular processes in general and may be used specifically in the design of new therapeutic agents with-for example, antitumorigenic properties.

University of Melbourne Department of Medicine,

P R GIBSON

The Royal Melbourne Hospital,

Victoria, 3050 Australia

1 Roediger WEW. The colonic epithelium in ulcerative colitis - an energy deficiency disease? Lancet 1980;ii:712-15.
2 Harig JM, Soergel KH, Komorowski RA, et al. Treatment of diversion colitis with short-chain fatty acid irrigation. $N$ Engl f Med 1989;320:23-8.

3 Kripke SA, Fox AD, Berman JL, et al. Stimulation of intestinal mucosal growth with intracolonic infusion of short chain fatty acids. F Parent Enteral Nutr 1989;13:109-16.

4 Singh B, Halestrap AP, Paraskeva C. Butyrate can act as a stimulator of growth or inducer of apoptosis in human colonic epithelial cell lines depending on the presence of alternative energy sources. Carcinogenesis depending on the

5 Rickard K, Gibson PR, Young GP, et al. Activation of protein kinase C augments butyrate-induced differentiation in colonic epithelial cells in vitro. Carcinogenesis 1999;20:977-84.

6 DePinho RA. The cancer-chromatin connection. Nature 1998;391:535-6.

7 Dangond F, Gullans SR. Differential expression of human histone deactylase mRNAs in response to immune cell apoptosis induction by trichostatin A and butyrate. Biochem Biophys Res Commun 1998;247:833-7.

8 Cuisset L, Tichonicky L, Delpech M. A protein phosphatase is involved in the inhibition of histone deactylation by sodium butyrate. Biochem Biophys Res Commun 1998;246:760-4.

\section{Think cytokines before you drink}

Conditions which arise from single gene defects demonstrate a simple mendelian mode of inheritance. There are however a large number of common conditions in which genetic factors are thought to be involved but are not clearly passed from one individual to another and merely cluster in families. Such observations raise a number of questions including: what is the size of the genetic contribution to the disorder and how might the susceptibility gene(s) involved in the development of the condition be identified?

The National Academy of Sciences-National Research Council twin registry of almost 16000 twin pairs reported concordance rates for cirrhosis of $16.9 \%$ in monozygotic twins and $5.3 \%$ in dizygotic twins, implying a genetic predisposition to this complication of alcohol abuse. ${ }^{1} \mathrm{Al}-$ though it is possible that twins share not only genes but a similar environment, with greater (in utero) environmental sharing in monozygotic twins, there is increasing epidemiological $^{2}$ and laboratory ${ }^{3}$ evidence to support a genetic basis to familial clustering. However, the concordance rate for alcoholic liver disease in monozygotic twins falls well below $100 \%$, highlighting the role of environmental as well as genetic factors.

The magnitude of the genetic contribution to disease is not easy to assess in common disorders. The overall recurrence risk ratio, or lambda $(\lambda)$ s of Risch, ${ }^{4}$ which is the ratio of the risk of a second sibling developing a disease where the first sibling already has the disease divided by the population prevalence of the disease, can be used to estimate the degree of familial clustering and, therefore, quantify the genetic risk. A $\lambda \mathrm{s}$ of 1 implies no genetic contribution whereas in single gene defects such as haemophilia, the ratio exceeds 1000 . Common complex disorders including for example diabetes, epilepsy, psoriasis, and multiple sclerosis, where both genetic and environmental effects are contributing to disease development, demonstrate a $\lambda$ s of $<20$. The ratio for alcoholic cirrhosis is not known although it is likely to be of a similar order of magnitude. In general terms the smaller the $\lambda s$ the harder it is to identify not only the gene(s) involved in the disease but also separate the primary aetiological mutation within the gene from the many polymorphisms which exist within the gene region.

In the search for genetic susceptibility loci for common complex disorders, three main approaches have been used: population based case control studies, intrafamilial association studies, and classical linkage analysis. Case control studies are a sensitive method of gene detection and the collection of subjects is relatively straightforward. However, they require a candidate gene to be studied and are prone to the generation of inconsistent results due to false positives arising from population mismatch or as a result of a random chance event because of small numbers. Intrafamilial association studies also require a candidate gene but eliminate false positives which arise from population mismatch by examining transmission of susceptibility alleles from parents to disease affected offspring. ${ }^{5}$ As parental DNA is required in addition to that of the index case, these studies are not as simple to conduct as case control studies. Linkage analysis is a powerful but complex tool for detecting major genes and is generally used as a means of systematically screening the genome in family based data sets. Its use is restricted because the collection of sib-pairs and multiple family members may be extremely difficult (as is the case with alcoholic liver disease) and its ability to detect genes of "modest" effect has proved limited. ${ }^{6}$

Candidate gene studies in alcoholic liver disease have recently moved from genes encoding ethanol metabolism to immune response genes because of their potential role in disease pathogenesis. In this issue, Grove et al (see page 540) have performed a population based, case control study to determine if the polymorphism associated with low interleukin 10 (IL-10) production is associated with and, therefore, a risk factor for advanced alcoholic liver disease (AALD). The A allele of the single nucleotide polymorphism (SNP) at position -627 (C-A) in the promoter of the $I L-10$ gene was found to be more common in patients with AALD compared with either normal controls or heavy drinkers with no evidence of liver disease, resulting in a relative risk for the development of AALD of 2.04 and 1.76, respectively. This paper is the first report of an association between polymorphism of the cytokine gene $I L-10$ and AALD. Although it is not possible with this type of study to determine if the associated allele is the primary disease causing mutation or is acting as a marker in linkage disequilibrium with a nearby as yet unknown aetioloigcal mutation, even in a neighbouring gene, there is good circumstantial functional evidence to implicate the $I L-10$ gene in AALD. The data presented are convincing, in that the total number of subjects used in the study was significant (621) and two sets of appropriate controls were used as comparators. The potential pitfalls of the population based case control approach in general and those specific to AALD which may have resulted in false positive results are carefully discussed by the authors. As they point out, 
family based AALD data sets would be useful to exclude population mismatching but their collection would be problematic. Replication of the data of Grove et al in a further independent data set is however vital, even if this is in the form of a second population based case control study. While confirmatory findings in an ethnically distinct population as opposed to similar ethnicity would be desirable, this is not essential. Replication of these data would strengthen the argument for subsequent functional molecular biological studies to help "nail" the primary aetiological disease causing mutation.

As with the study of all complex diseases, establishment of large multiple independent data sets is vital in attempts at identifying susceptibility loci which are likely to be exerting small but clinically significant effects. It is unlikely that any one group will have sufficient numbers of patients to establish more than one data set and, therefore, working collaborations between groups should be encouraged. With the ongoing emergence of more detailed genetic maps, including the availability of in silico candidate SNP markers and the development of technology capable of performing large scale genotyping, those groups who have established large data sets will be the first to identify susceptibility genes.

S C L GOUGH

Department of Medicine,

University of Birmingham,

Birmingham Heartlands Hospital,

Bordesely Green East,

Birmingham B9 5SS, UK

Email:s.c.gough@bham.ac.uk

1 Reed T, Page WF, Viken RJ, Christian JC. Genetic predisposition to organspecific endpoints of alcoholism. Alcohol Clin Exp Res 1996;20:1528-33.

2 Ebers GC, Sadovnick AD, Risch NJ. A genetic basis for familial aggregation in multiple sclerosis. Canadian Collaborative Study Group. Nature 1995;377:150-1.

3 Todd JA. Genetic analysis of type 1 diabetes using whole genome approaches. Proc Natl Acad Sci USA 1995;92:8560-5.

4 Risch N. Linkage strategies for genetically complex traits. II. The power of affected relative pairs. Am f Hum Genet 1990;46:229-41.

5 Spielman RS, McGinnis RE, Ewens WJ. Transmission test for linkage disequilibrium: the insulin gene region and insulin-dependent diabetes mellitus (IDDM). Am f Hum Genet 1993;52:506-16.

6 Risch N, Merikangas K. The future of genetic studies of complex human diseases. Science 1996;273:1516-17.

\section{LKM antibody: getting in some target practice}

In the current issue of the journal (see page 553), Muratori et al provide convincing evidence that cytochrome P4502D6 (CYP2D6) is present on the liver cell plasma membrane. This finding has important implications because CYP2D6 is the main target of liver kidney microsomal antibody type 1 (LKM1). Not only is LKM1 the serological hallmark of autoimmune hepatitis type 2 but it is also found in up to $10 \%$ of patients with chronic hepatitis $\mathrm{C}$ virus (HCV) infection where it appears to single out patients experiencing serious side effects while receiving interferon treatment. ${ }^{1}$

The issue as to whether CYP2D6 is present on the plasma membrane was critically reviewed in $1993^{2}$ but was not resolved in view of the contrasting evidence at the time. Muratori et al have settled this matter by incubating LKM1 containing sera with live hepatocytes which can offer the external aspect of the plasma membrane to the antibody. The presence of hepatocyte bound antibody is then determined by addition of a second antibody emitting a green fluorescent signal. Lastly and crucially, an antibody specific for CYP2D6, and emitting a red fluorescent signal, is used to counterstain the hepatocytes. With this manoeuvre when "green" and "red" antibodies recognise the same structure, a yellow signal is generated. And, as the reader can verify on page 000 , yellow staining results from the experiment described above demonstrating elegantly that: (1) LKM1 positive sera react with the outer aspect of the plasma membrane; and (2) the target they recognise is CYP2D6.

In common with other liver cytochromes, CYP2D6 is mainly localised in the endoplasmic reticulum and sediments in the so-called "microsomal" fraction following differential ultracentrifugation of a liver homogenate. The microsomal fraction was used in the original absorption experiments ${ }^{3}$ aimed at identifying the antigen targeted by LKM1 (hence the L and M of the acronym). The ability of cytochromes to migrate to the plasma membrane has been documented by the studies of Robin et al. ${ }^{4}$ There is an extensive flow of vesicles from the endoplasmic reticulum, in which CYPs are anchored through their N-terminal end, to the Golgi apparatus, and then along the microtubules to the plasma membrane. ${ }^{5}$ Pulse chase labelling experiments show that the bulk of CYPs reside in the endoplasmic reticulum where the label persists for several hours, while about $10 \%$ is present in the plasma membrane where the label lasts for minutes, indicating fast turnover of the enzymes in this location, the probable result of rapid endocytotic membrane internalisation. ${ }^{4}$

The presence of an autoantibody target on the plasma membrane has pathogenic implications. LKM1 now ceases to be just a marker of disease ${ }^{1}$ and acquires damaging connotations. Thus the association of LKM1 with a particularly aggressive form of autoimmune hepatitis, ${ }^{6}$ and with the severity of liver injury in LKM1 positive chronic HCV patients, ${ }^{7}$ is now seen in a new light. As indeed is the fact that LKM1 singles out among HCV positive patients a group prone to develop severe side effects during interferon treatment.

The question as to how CYP2D6 becomes an autoantigen remains unresolved. CYPs represent an enzymatic system evolved as a defence mechanism against xenobiotics, such as drugs, metals, industrial and naturally occurring chemicals. In this capacity CYP2D6 metabolises debrisoquine and several other drugs. The detoxifying function of cytochromes may provide a clue as to how they can become autoantigens. ${ }^{8}$ In the line of service, these enzymes generate reactive metabolites capable of altering the shape of the molecule to a degree not tolerated by the immune system. The immune response may then also turn against the native form of the enzyme. In an alternative scenario, CYPs may become autoantigens as a consequence of molecular mimicry and cross reactive immunity. ${ }^{9}$ CYP2D6 shares sequences in common with viruses such as herpes viruses, including CMV, and with HCV, hence the suggestion by Manns and colleagues ${ }^{10}$ that cross reactive immunity explains the presence of LKM1 in HCV infection. The question of whether CYP2D6 becomes an autoantigen for its detoxifying functions or because it is targeted by a "friendly immunological fire", a fire originally intended at eliminating a pathogen that too closely resembles sequences of the enzyme, will be addressed in the months and years to come. 
Institute of Hepatology,

University College London Medical School,

69-75 Chenies Merws,

London WC1E 6HX, UK

Email: D.Vergani@ucl.ac.uk

1 Vergani D. NOSA in HCV infection: markers or makers of disease? Gut 1999;45:328-9

2 Vergani D, Mieli-Vergani G. Type II autoimmune hepatitis: the conundrum of cytochrome P450IID6. Clin Exp Immunol 1993;92:367-8.

3 Rizzetto M, Swana G, Doniach D. Microsomal antibodies in active chronic hepatitis and other disorders. Clin Exp Immunol 1973;15:331-44.

4 Robin MA, Le Roy M, Descatoire V, Pessayre D. Plasma membrane cytochromes $\mathrm{P} 450$ as neoantigens and autoimmune targets in druginduced hepatitis. F Hepatol 1997;26 (suppl 1):23-30
D VERGANI

$$
\begin{aligned}
& \text { vesicular route to the plasma membrane in cultured rat hepatocytes. } \\
& \text { Gastroenterology 1995;108:1110-23. } \\
& 6 \text { Mieli-Vergani G, Vergani D. Immunological liver diseases in children. Semin } \\
& \text { Liver Dis 1998;18:271-9. } \\
& 7 \text { Giostra F, Manzin A, Lenzi M, et al. Low hepatitis C viremia levels in } \\
& \text { patients with anti-liver/kidney microsomal antibody type } 1 \text { positive chronic } \\
& \text { hepatitis. F Hepatol 1996;25:433-8. } \\
& 8 \text { Griem P, Wulferink M, Sachs B, Gonzalez JB, Gleichmann E. Allergic and } \\
& \text { autoimmune reactions to xenobiotics: how do they arise? Immunol Today } \\
& \text { 1998;19:133-41. } \\
& 9 \text { Oldstone MB. Molecular mimicry and autoimmune disease (published } \\
& \text { erratum appears in Cell 1987;51:878). Cell 1987;50:819-20. } \\
& 10 \text { Manns MP, Griffin KJ, Sullivan KF, Johnson EF. LKM-1 autoantibodies } \\
& \text { recognize a short linear sequence in P450IID6, a cytochrome P-450 } \\
& \text { monooxygenase. } \mathcal{F} \text { Clin Invest 1991;88:1370-8. }
\end{aligned}
$$

Robin MA, Maratrat M, Loeper J, et al. Cytochrome P4502B follows a

\section{Combination therapy of hepatitis B}

There are now two licensed therapies for chronic hepatitis B: interferon $\alpha$ and lamivudine. Interferon $\alpha$ was first shown to have activity against hepatitis B in $1976,{ }^{1}$ but was not formally approved for use in chronic hepatitis B until 1992. The currently recommended regimen for interferon is 5 million units (mu) given daily or $10 \mathrm{mu}$ given three times a week by subcutaneous (sc) injection for four to six months. This regimen results in long term beneficial responses in roughly $33 \%$ of patients. ${ }^{2}{ }^{3}$

Lamivudine was first shown to have activity against hepatitis B virus (HBV) in $1992^{4}$ and was approved for use in chronic hepatitis B in 1998. The currently recommended regimen for lamivudine is $100 \mathrm{mg}$ given daily by mouth for one year. This regimen results in beneficial responses in $16-20 \%$ of patients with typical chronic hepatitis B. ${ }^{5-7}$

Thus, there are now two choices for therapy. Which should be used? Which should be used first? Should the second be tried if the first fails? What about combining the two? These are simple questions, but they do not have simple answers.

In this issue (see page 562), Schalm and collaborators report results of a large, multinational, randomised, double blind, placebo controlled trial of lamivudine, interferon $\alpha$ and the combination of both in 230 patients with typical chronic hepatitis $\mathrm{B}$. This trial was one of the largest studies ever conducted in hepatitis B and was large enough to have answered the questions posed above, at least in part. In the study, $18 \%$ of patients receiving lamivudine alone, $19 \%$ receiving interferon alone and $29 \%$ receiving the combination had a beneficial response, the higher response rate with combination therapy being of borderline statistical significance. Side effects from the combination were no greater than those with interferon alone. These results suggest that the combination of lamivudine and interferon $\alpha$ is the optimal initial therapy of this disease.

Before such recommendations are embraced, however, a closer look at this study is needed. Unfortunately, the study suffered in several regards: in design, conduct and analysis.

The design of this three arm study was complex. The first two arms were standard. One group received the recommended regimen of lamivudine and the second, the recommended regimen of interferon. The combination group, however, received an unusual and irregular regimen of both: lamivudine was given for six months only and interferon was started late, two months after initiating lamivudine therapy. The "lead in" phase of lamivudine before combination therapy was done because of previous studies suggesting that interferon is more effective in patients with lower levels of viral DNA. ${ }^{2}{ }^{3}$ The difficulty is that interferon is also more effective in patients with higher serum aminotransferase activities, and lamivudine therapy often lowers serum enzyme activities. Furthermore, the complex regimen made the blinding of placebo treatment difficult.

Another major problem with the design was the differences in the timing of the end point evaluation in relation to therapy. Thus, the end points were measured in patients receiving lamivudine while they were still on treatment, but in those receiving interferon or the combination at a point six months after stopping therapy. This variability complicated the comparison of end points among groups. Thus, at the one year point, HBV DNA levels, aminotransferases and liver histology may well have been affected by the continuation of lamivudine therapy. Furthermore, scant information was given about follow up of treated patients after stopping lamivudine. Of course, it is difficult to compare therapies if the durations are different between groups (and this problem has plagued other studies of therapy of viral hepatitis). ${ }^{8}$ This discrepancy can only be overcome by adequate follow up, which would be at least 12 months after stopping lamivudine and, therefore, 18 months after stopping interferon with or without lamivudine.

The conduct of the trial was also problematic. The number of protocol violations was high, occurring in 50 of the 230 randomised patients. These violations consisted of dropouts, errors in randomisation, errors in assignment of inclusion and exclusion criteria, use of other antiviral agents during the study, and other problems. The number of protocol violations may have been because of the complexity of the design, lack of acceptance of a placebo control, the diversity of the therapies, and the large number of geographically dispersed centres involved.

The analysis and presentation of results of this trial were also complex and difficult. Results were presented using five different populations of patients enrolled: the total "as treated" population of 230 patients, the "intention to treat" population of 226 patients, the "per protocol" population of 180 patients (minus the violations), the population of 212 patients who reached the 12 month point for analysis, and the population of 165 patients reaching the 15 month point. The shifting denominator of number of patients makes it difficult to state what the response rates were in terms that are understandable clinically. It is also difficult to say which group should have been used. For instance, in the "per protocol" analysis, the response rate to combination therapy was $36 \%$, quite a bit better and supportive of combination therapy than the $22 \%$ for interferon alone and $19 \%$ for lamivudine alone. 
A final critical issue in analysis was the end point used to define benefit. A "response" was defined as seroconversion from $\mathrm{HBeAg}$ to anti-HBe (and absence of HBV DNA by hybridisation testing) by the 12 month point. This end point differs from most trials of antiviral therapy of hepatitis $\mathrm{B}$ which used the loss of $\mathrm{HBeAg}$ (with or without development of anti-HBe) as an end point. ${ }^{2}{ }^{3}$ Using loss of $\mathrm{HBeAg}$ as a definition of response (from table 2 and the population of 214 patients), $29 \%$ of lamivudine treated, $36 \%$ of interferon treated, and $51 \%$ of combination treated patients responded. Loss of $\mathrm{HBeAg}$ is a surrogate marker that has been found to be reasonably reliable in predicting a long term remission after interferon therapy of chronic hepatitis B as shown in several long term follow up studies. ${ }^{9}{ }^{10}$ Whether loss of HBeAg or seroconversion to anti-HBe is a reliable surrogate marker for a sustained remission after nucleoside analogue therapy is unknown. In this study, nine of 11 patients who lost $\mathrm{HBeAg}$ during lamivudine therapy remained $\mathrm{HBeAg}$ negative three months later (data from panel A of figure 2 are, however, incompatible with this statement from the text). This number represents a $18 \%$ relapse rate within three months of stopping therapy, quite high when one considers that this is a chronic, often life-long disease.

Despite these shortcomings, this trial provides a large amount of important information about therapy of hepatitis B. Additional information would have been helpful, especially with regard to the effect of the lead in phase on HBV DNA and alanine aminotransferase activities and whether responses were sustained long term. Additionally, use of a more sensitive assay for HBV DNA levels (such as PCR) might have helped to resolve differences in the three groups.

What can be recommended from this study? Another study, certainly. But if a clinician plans to treat patients with hepatitis $\mathrm{B}$, a combination approach is reasonable and should probably employ a conventional regimen of both agents, starting both interferon and lamivudine at the same time, continuing interferon for four months and lamivudine for 12 months. In clinical practice, as in clinical research studies, long term follow up is needed of all treated patients to document absence of reactivation and continued resolution of disease activity.
Antiviral therapy of hepatitis B has entered adolescence, the awkward age-restive and resistant to management. The availability of two agents to treat this disease has provided more opportunities, but with it goes more responsibilities and more difficult choices. A safe and secure maturity will probably require a third or fourth agent active against this disease.

E DOO

Liver Diseases Section,

Digestive Diseases Branch,

National Institute of Diabetes and Digestive and Kidney Diseases,

Building 10, Room 9B06,

NIH, Bethesda, MD 20892, USA

Email:DooE@intra.niddk.nih.gov

Liver Diseases Section,

Digestive Diseases Branch,

National Institute of Diabetes and Digestive and Kidney Diseases,

Building 31, Room $9 A 23$,

NIH, Bethesda, MD 20892, USA

Email:Hoofnaglef@extra.niddk.nih.gov

Conflict of Interest Statement: The authors are associate investigators in a clinical trial of lamivudine therapy at the National Institutes of Health supported in part by Glaxo-Wellcome, but neither has received direct funding, salary or honoraria from Glaxo-Wellcome.

1 Greenberg HB, Pollard RB, Lutwick LI, et al. Effect of human leucocyte interferon on hepatitis B virus infection in patients with chronic active interferon on hepatitis B virus infection

2 Hoofnagle JH, Di Bisceglie AM. Treatment of chronic viral hepatitis. N Engl f Med 1997;336:347-56.

3 Wong JB, Koff RS, Tine F, et al. Cost-effectiveness of interferon alpha- $2 \mathrm{~b}$ treatment for hepatitis B e-antigen positive chronic hepatitis B. Ann Intern Med 1995;122:664-75

4 Chang CN, Skalski V, Zhou JH, et al. Biochemical pharmacology of (+)-2',3'-dideoxy-3'-thiacytidine and (-)-2',3'-dideoxy-3'-thiacytidine as antihepatitis B-virus agents. F Biol Chem 1992;267:22414-20.

5 Lai CL, Chien RN, Leung NWY, et al. A one-year trial of lamivudine for chronic hepatitis B. N Engl f Med 1998;339:61-8.

6 Dienstag JL, Perrillo RP, Schiff ER, et al. A preliminary trial of lamivudine for chronic hepatitis B infection. N Engl f Med 1995;333:1657-61.

7 Mutimer D, Naoumov N, Honkoop P, et al. Combination alpha-interferon and lamivudine therapy for alpha-interferon-resistant chronic hepatitis $\mathrm{B}$ and lamivudine therapy for alpha-interferon-resistant chro

8 Poynard T, Bedossa P, Chevallier M, et al. A comparison of three interferon Poynard T, Bedossa $\mathrm{P}$, Chevallier $\mathrm{M}$, et al. A comparison of three interferon alfa-2b regimens for the long-term treatment
hepatitis. N Engl f Med 1995;332:1457-62.

9 Niederau C, Heintges T, Lange S, et al. Long-term follow-up of HBeAg positive patients with chronic active hepatitis after $\alpha$-interferon therapy. $N$ Engl F Med 1996;334:1422-7.

10 Lau DT-Y, Everhart J, Kleiner DE, et al. Long-term follow-up of patients with chronic hepatitis B treated with interferon alfa. Gastroenterology 1997; 113:1660-7. 